\title{
Plasma Catalase in Relation to Pain Following Midline Laparotomy: A Prospective Study of Patients with Benign Diseases and Patients with Cancer
}

\author{
IINA SAIMANEN ${ }^{1}$, JARI KÄRKKÄINEN ${ }^{1}$, TUOMAS SELANDER ${ }^{2}$, MARTIN PURDY ${ }^{1}$, \\ MERJA KOKKI ${ }^{3}$, HANNU KOKKI ${ }^{3}$ and MATTI ESKELINEN ${ }^{1}$ \\ ${ }^{1}$ Department of Surgery, Kuopio University Hospital and School of Medicine, \\ University of Eastern Finland, Kuopio, Finland; \\ ${ }^{2}$ Science Service Center, Kuopio University Hospital, Kuopio, Finland; \\ ${ }^{3}$ Department of Anaesthesia and Operative Services, Kuopio University Hospital and School of Medicine, \\ University of Eastern Finland, Kиорio, Kuоpio, Finland
}

\begin{abstract}
Background/Aim: The relationship of plasma concentrations of the oxidative stress biomarker catalase with pain on numeric rating scale at rest (NRSr) and under wound pressure 24 hours postoperatively (NRSp) in midline laparotomy patients with rectus sheath block (RSB) analgesia are unknown. Our original hypothesis was that RSB analgesia might reduce postoperative pain. Patients and Methods: Initially, 56 patients were randomized to four groups: control group $(n=12)$, single-dose $(n=16)$, repeated-dose $(n=12)$ and continuous infusion ( $n=16)$ RSB analgesia groups. The plasma concentrations of catalase were measured immediately before, immediately after and 24 hours after surgery. The pain at rest and under pressure were scored on an 11-point numeric rating scale 24 hours postoperatively $\left(N R S_{r}\right.$ and $N R S_{p}$; 0: no pain; 10: worst pain). Results: The median plasma concentration of catalase increased immediately after surgery $(p=0.007)$, but then decreased 24 hours postoperatively compared with immediately after surgery $(p<0.001)$. The control group and the RSB groups did not differ in individual $N R S_{r}$ and $N R S_{p}$ values following surgery. Scatter plots of the plasma catalase versus superoxide dismutase concentrations were positively correlated in the patients $(r=0.314, p<0.001)$. In addition, scatter plots of the individual $N R S_{r}$ and $N R S_{p}$ values versus plasma values of catalase were inversely correlated in patients $(r=-0.221, p=0.03$ and $r=-0.238, p=0.02$, respectively).
\end{abstract}

Correspondence to: Matti Eskelinen, MD, Ph.D., School of Medicine, University of Eastern Finland, P.O. Box 100, FI-70029 KYS, Finland. Tel: +358 17173311, Fax: +358 17172611, GSM: +358400969444, e-mail: matti.eskelinen@kuh.fi

Key Words: Benign disease, plasma catalase, oxidative response, pain following surgery.
Conclusion: The RSB analgesia does not reduce postoperative pain. Plasma catalase levels and pain following surgery are significantly correlated in patients regardless of disease type.

In a Swedish study on gastrointestinal surgery, the proportion of laparoscopy in minor surgery was $47-97 \%$ during 2012 2014 and the four most common procedures were appendectomy, cholecystectomy, fundoplication and bariatric operation. However, the proportion of laparoscopy in major resection surgery was rather low at $4-10 \%$ including esophago-gastric, colorectal, liver and pancreatic operations (1). In addition, midline laparotomy is still needed in emergency abdominal operations, where it reveals the whole peritoneal cavity and allows all the intra-abdominal organs to be handled despite adhesions, serious bleeding or peritonitis (2). In the midline laparotomy, patients need proper analgesia following surgery and different pain treatment modalities have to be assessed and it is also very important to predict higher analgesia requirements for these patients. Our previous article reported a statistically significant inverse correlation between the plasma catalase concentration 6 hours postoperatively and the individual pain assessments with the numeric rating scale (NRS) 24 hours postoperatively in patients with cholelithiasis (3). Yiannakopoulou et al. reviewed the clinical trials that investigated oxidative stress in open versus laparoscopic surgery (4). They found two trials $(5,6)$ on plasma levels of catalase in oxidative stress response to surgery and their systematic review aimed to investigate the impact of surgical trauma on oxidative stress.

Mitochondria are a source of reactive oxygen species (ROS), which includes non-radicals such as hydrogen peroxide and free radicals such as hydroxyl radical, peroxyl radical and superoxide radical $(7,8)$. Intracellular accumulation of ROS causes oxidative stress in many acute 


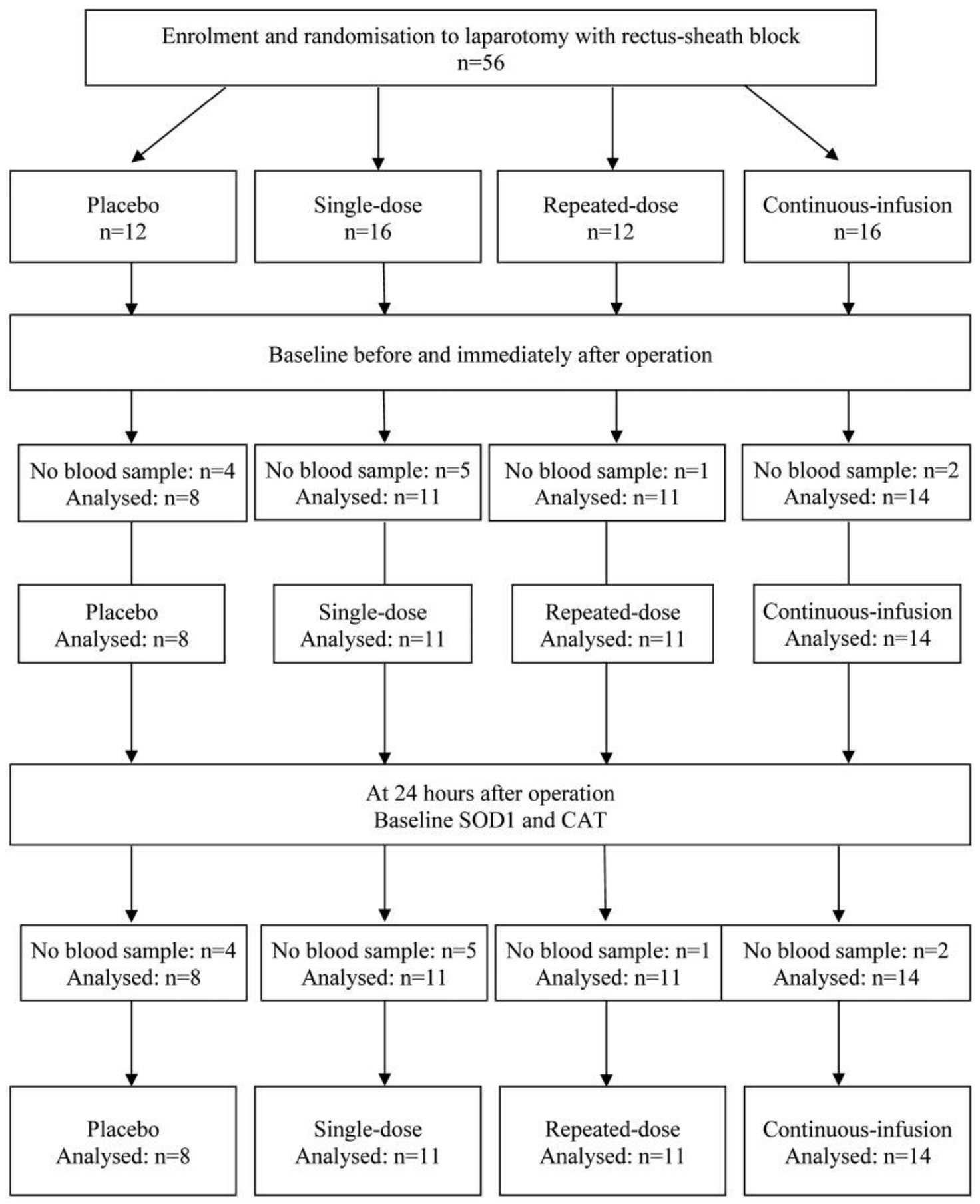

Figure 1. Design of the study as flowchart.

and chronic diseases, including sepsis (9), cancer and liver disease $(10,11)$. Superoxide dismutase (SOD) and catalase have been used as indicators of oxidative stress and their increased activity seems to be a protective response against ROS (11). SOD catalyses the dismutation of superoxide anion into hydrogen peroxide. Catalase is an enzyme responsible for the conversion of hydrogen peroxide to water and oxygen. High catalase levels would be a response to increased SOD activity. The effects of catalase on a biomarker of ROS in response to midline laparotomy with rectus sheath block 
Table I. The midline laparotomy patients' clinical data in the four study groups. Data are mean (standard deviation) or number of cases. The postoperative are shown as median and interquartile range (Kruskal-Wallis test).

\begin{tabular}{|c|c|c|c|c|c|}
\hline \multirow[b]{2}{*}{ Variable } & \multicolumn{5}{|c|}{ RSB group } \\
\hline & Control $n=8$ & Single $\mathrm{n}=11$ & Repeated $n=11$ & Continuous $n=14$ & $p$-Value \\
\hline Age, years & $62.6(14.3)$ & $60.8(12.6)$ & $63.3(10.8)$ & $58.0(10.1)$ & 0.74 \\
\hline Gender, male/female & $4 / 4$ & $4 / 7$ & $2 / 9$ & $2 / 12$ & 0.22 \\
\hline Height, $\mathrm{cm}$ & $166.6(8.6)$ & $168.4(7.9)$ & $165.7(7.2)$ & $164.3(6.6)$ & 0.62 \\
\hline Weight, kg & $78.6(11.8)$ & $83.7(12.8)$ & $67.8(13.7)$ & $68.8(10.6)$ & 0.007 \\
\hline BMI, $\mathrm{kg} / \mathrm{m}^{2}$ & $28.3(3.8)$ & $29.6(4.4)$ & $24.6(4.3)$ & $25.7(4.9)$ & 0.03 \\
\hline Time in the operative room, $\min$ & $229.4(113.4)$ & $274.9(148.4)$ & $235.7(112.0)$ & $279.7(178.5)$ & 0.85 \\
\hline Operative time, $\min$ & $209.6(141.2)$ & $221.8(156.4)$ & $154.4(95.0)$ & $253.3(168.9)$ & 0.55 \\
\hline Perioperative bleed, $\mathrm{ml}$ & $696(741)$ & $822(906)$ & $697(967)$ & $1340(928)$ & 0.31 \\
\hline ASA $1 / 2 / 3 / 4$ & $0 / 6 / 2 / 0$ & $0 / 7 / 3 / 1$ & $0 / 5 / 6 / 0$ & $2 / 7 / 5 / 0$ & 0.43 \\
\hline $\mathrm{NRS}_{\mathrm{r}}$ & $2(1-6)$ & $2(0-4)$ & $2(0-3)$ & $1(0-4)$ & 0.41 \\
\hline $\mathrm{NRS}_{\mathrm{p}}$ & $5(3-9)$ & $5(1-9)$ & $3.5(1-7)$ & $4(0-7)$ & 0.42 \\
\hline Length of the skin incision(s), mm & $27.2(6.6)$ & $24.4(7.8)$ & $24.2(7.9)$ & $29.7(7.3)$ & 0.31 \\
\hline \multicolumn{6}{|l|}{ Type of disease } \\
\hline Benign $(n=15)$ & 1 & 3 & 5 & 6 & 0.32 \\
\hline GI cancer $(n=9)$ & 3 & 2 & 2 & 2 & \\
\hline Gyn cancer $(n=17)$ & 3 & 4 & 4 & 6 & \\
\hline Other cancer $(n=3)$ & 1 & 2 & 0 & 0 & \\
\hline
\end{tabular}

GI: Gastrointestinal tract; Gy: gynaecological; BMI: body mass index; ASA: American Society of Anesthesiologists physical status score; NRS:11point numeric rating scale $24 \mathrm{~h}$ after surgery; NRSr: NRS at rest; NRSp: NRS under $2 \mathrm{~kg}$ pressure to the wound area; RSB: rectal sheath block.

(RSB) analgesia is unknown. Therefore, our main aim was to study the effect of different modalities of RSB analgesia in relation to plasma catalase concentrations and postoperative pain with the hypothesis was that RSB analgesia might reduce the impact of surgical oxidative stress and postoperative pain in patients with benign diseases and those with cancer.

\section{Patients and Methods}

The study was approved by the Ethics Committee of Kuopio University Hospital District, Finland (DNRO 120/2011, November 11,2011 ) and was registered in the EudraCT database (EudraCT number 2011-005136-25) and in the ClinicalTrials.gov database (ClinicalTrials.gov Identifier: NCT02869841). It was conducted in accordance with the Declaration of Helsinki. Participants gave written consent after receiving verbal and written information.

Operations were carried out at Kuopio University Hospital, Kuopio, Finland between 2012 and 2015. The CONSORT flowchart of the study is presented in Figure 1. The study design was a prospective, randomized, clinical trial with four parallel groups. The patients with midline laparotomy were randomized into the control group or into one of the three active groups: single-dose, repeateddose or continuous infusion RSB analgesia groups. The study patients had intravenous oxycodone pumps as patient-controlled analgesia (PCA). The randomization list was generated by computer (www.randomization.com) and a sealed enveloped method was used for blinding and randomisation was done preoperatively.

The primary outcome measure here was the plasma concentration of catalase measured at three time points with high-sensitivity assays: before, immediately after and 24 hours after surgery. Pain
Table II. The postoperative alteration of plasma superoxide dismutase 1 (SOD1) and catalase (CAT) concentrations in all groups combined (all patients). Plasma concentrations were measured before (PRE), immediately after (POP1) and 24 hours after (POP2) surgery. Median (interquartile range) concentrations are shown.

\begin{tabular}{lcc}
\hline & All patients & $p$-Value* \\
\hline SOD1 $(\mathrm{pg} / \mathrm{ml})$ & & \\
PRE & $164.0(114-213)$ & \\
POP1 & $185.0(137-301)$ & $0.007 \mathrm{vs.}$ PRE \\
POP2 & $105.0(86-176)$ & $<0.001 \mathrm{vs.}$ POP1 \\
CAT $(\mathrm{pg} / \mathrm{ml})$ & & \\
PRE & $0.66(0.48-1.17)$ & \\
POP1 & $1.39(0.84-2.43)$ & $<0.001 \mathrm{vs.}$ PRE \\
POP2 & $1.03(0.70-1.57)$ & $0.001 \mathrm{vs}$. POP1 \\
\hline
\end{tabular}

*Wilcoxon signed-rank test.

was assessed at 24 hours following surgery using a 11-point NRS (NRS: 0: no pain; 10: worst pain) at rest (NRSr) and at using $2 \mathrm{~kg}$ pressure on the wound area (NRSp).

All RSB procedures were performed by an experienced surgeon in the operating room before wound closure. The study protocol with the exclusion criteria and informed consent were fully described in our earlier original work $(2,12-15)$.

EDTA-blood samples were taken at the prespecified timepoints and centrifuged at $1,000 \times g$ for $15 \mathrm{~min}$. Plasma was separated and stored frozen at $-70^{\circ} \mathrm{C}$ until analysed. The plasma SOD1 assays were performed using sandwich-type ELISA methods from 
Table III. The plasma superoxide dismutase 1 (SOD1) and catalase (CAT) concentrations in the in the four study groups. Plasma concentrations were measured before (PRE), immediately after (POP1) and 24 hours after (POP2) surgery. Median (interquartile range) concentrations are shown.

\begin{tabular}{|c|c|c|c|c|c|}
\hline \multirow[b]{2}{*}{ Marker } & \multicolumn{5}{|c|}{ RSB group } \\
\hline & Control & Single & Repeated & Continuous & $p$-Value* \\
\hline \multicolumn{6}{|c|}{ SOD1 (pg/ml) } \\
\hline PRE & $150(101-288)$ & $146(96-287)$ & $171(113-216)$ & $168(128-199)$ & 0.922 \\
\hline POP1 & $281(144-522)$ & $160(101-305)$ & $184(123-275)$ & $243(151-292)$ & 0.392 \\
\hline POP2 & $119(93-202)$ & $87(59-105)$ & $116(88-171)$ & $167(94-217)$ & 0.138 \\
\hline \multicolumn{6}{|c|}{ CAT $(\mathrm{pg} / \mathrm{ml})$} \\
\hline PRE & $0.95(0.67-2.36)$ & $0.74(0.45-1.58)$ & $0.61(0.41-1.09)$ & $0.61(0.43-1.17)$ & 0.407 \\
\hline POP1 & $1.36(0.89-3.71)$ & $1.89(0.78-2.93)$ & $1.53(0.78-2.17)$ & $1.39(0.84-2.03)$ & 0.907 \\
\hline POP2 & $1.17(0.58-1.65)$ & $0.86(0.60-1.73)$ & $1.15(0.77-1.50)$ & $1.06(0.83-1.92)$ & 0.891 \\
\hline
\end{tabular}

*Kruskal-Wallis test.

BioVendor Cu/Zn SOD ELISA Kit (Brno, Czech Republic). The plasma catalase assays were performed using an enzyme-linked immunosorbent assay ELISA Kit (Cloud-Clone Corporation, Katy, TX, USA). The manufacturer's intra-assay and the inter-assay Cvs were $<10 \%$ and $<12 \%$, respectively.

The data were entered and analyzed with a statistical software program (IBM SPSS Statistics 24.0; IBM, Armonk, NY, USA). Differences in baseline characteristics between groups were tested by Fisher's exact test and in the case of continuous data, the analysis was performed by independent samples $t$-test. Group differences at three time points were tested by the Wilcoxon signedrank test and the Kruskal-Wallis test. The plasma catalase concentrations and differences in patients with benign diseases and cancer were tested by the Mann-Whitney $U$-test. The results of the laboratory measurements are presented as median values with interquartile range as distributions were right-skewed. A two-sided $p$-value of less than 0.05 was considered statistically significant. Pearson's method was used to test for correlation of individual catalase versus SOD1 concentrations and for catalase concentrations versus individual NRS at rest and under wound pressure.

\section{Results}

The study group consisted of 15 patients with benign disease, nine patients with gastrointestinal cancer, 17 patients with gynaecological cancer and three patients with other malignancy (Table I). The clinical data of midline laparotomy patients with benign disease and cancer were quite similar in the four study groups although patients body weights and body mass index were significantly higher in the single-dose and control groups than in the two other study groups ( $p=0.007$ and $p=0.03$, respectively, Table I).

The control group and the RSB groups did not differ in individual $\mathrm{NRS}_{\mathrm{r}}$ and $\mathrm{NRS}_{\mathrm{p}}$ values following surgery (Table I). The median plasma catalase and SOD1 concentrations immediately after surgery were statistically highly significantly increased (both $p<0.001$, Table II). The concentration of catalase

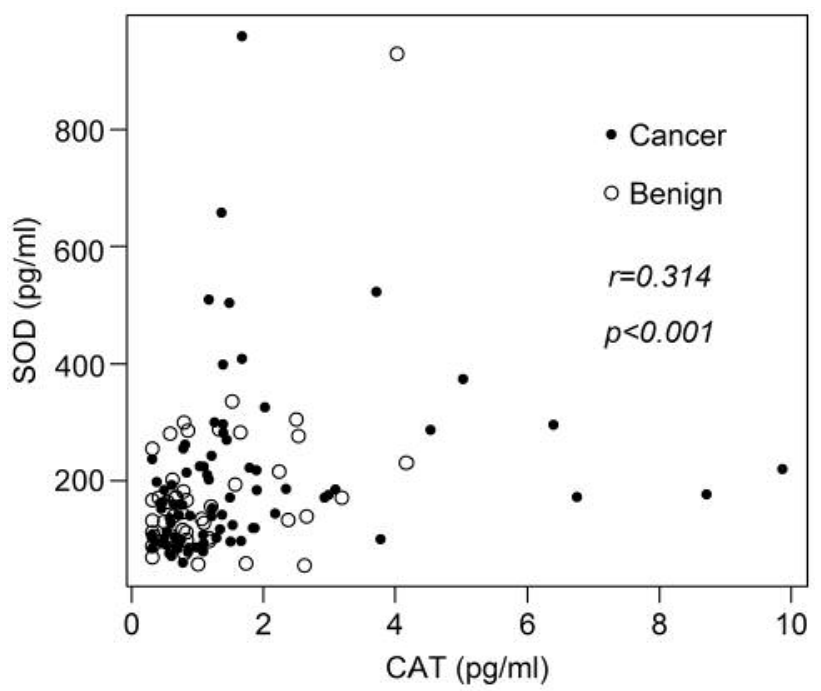

Figure 2. Scatter plots of the plasma catalase (CAT) concentrations versus superoxide dismutase (SOD1) concentrations in patients with benign disease and cancer $(r=0.314, p<0.001)$.

then decreased at 24 hours postoperatively ( $p=0.001$, Table II). However, no differences were detected in the catalase values between the control and the three active groups preoperatively and after surgery. The patients in the control group had a trend for higher median catalase level preoperatively compared to the single dose, repeated dose and continuous dose groups (Table III). The median plasma concentrations of catalase did not differ significantly between the patients with benign disease and those with cancer, neither pre- nor postoperatively, however (Table IV). Patients in the cancer group had a trend for higher median catalase concentration postoperatively compared to the patients with benign disease (Table IV). 

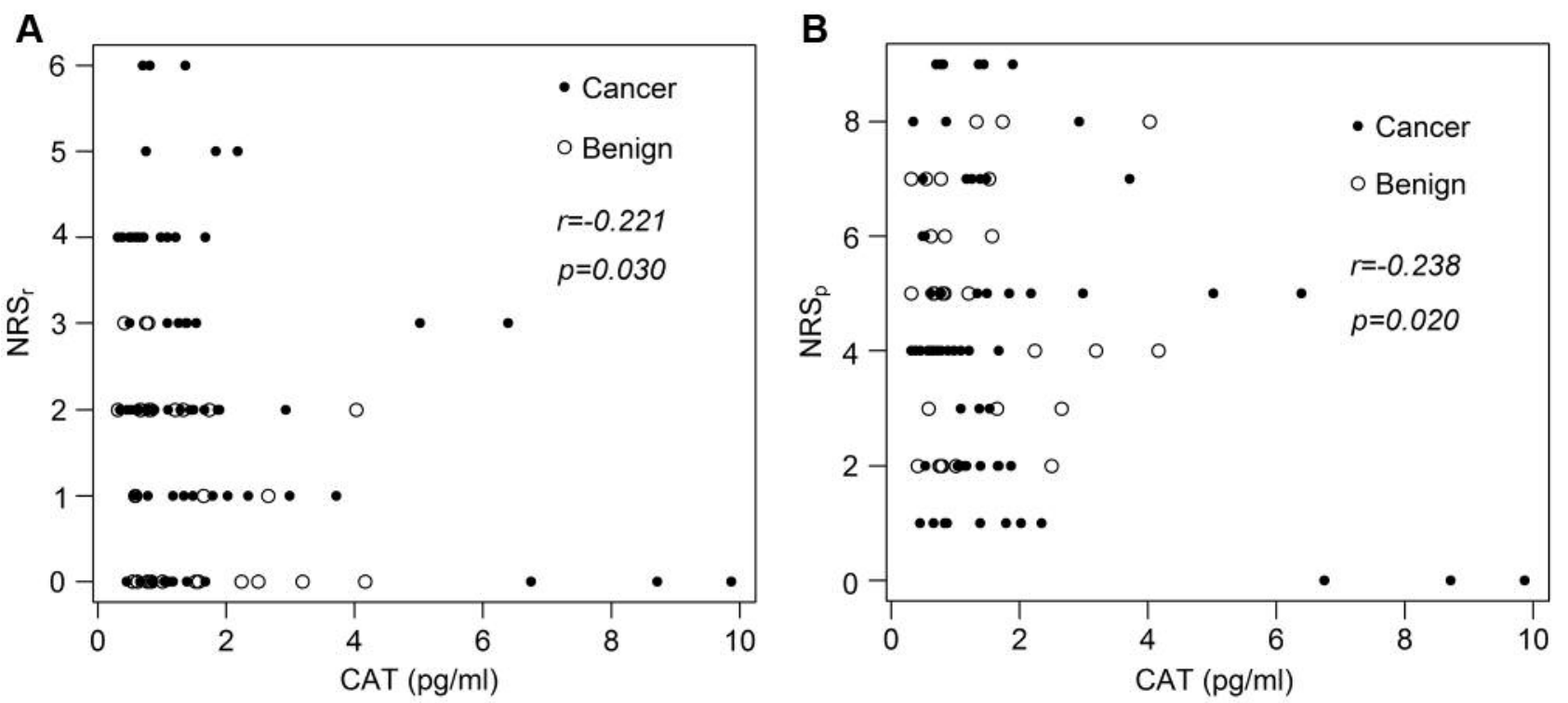

Figure 3. Scatter plots of the individual postoperative scores on an 11-point numeric rating scale: A: at rest (NRS $\left.S_{r}\right)$ and B: under $2 \mathrm{~kg}$ pressure to the wound area $\left(N R S_{p}\right)$ versus plasma concentrations of catalase $(C A T)$ in patients with benign disease and cancer.

Scatter plots of the plasma catalase values versus SOD1 concentrations for the whole patient cohort were significantly weakly positively correlated $(\mathrm{r}=0.314, p<0.001$, Figure 2$)$. In addition, scatter plots showed $\mathrm{NRS}_{\mathrm{r}}$ and $\mathrm{NRS}_{\mathrm{p}}$ (Figure 3) values were significantly weakly inversely correlated with plasma concentrations of catalase in patients $(\mathrm{r}=-0.221$, $p=0.03$ and $\mathrm{r}=-0.238, p=0.02$, respectively).

\section{Discussion}

Our original hypothesis was that RSB analgesia might reduce postoperative pain. However, the control group and the RSB groups did not differ in individual NRSr and NRSp values following surgery. Therefore, this result is disappointing for our working hypothesis, it seems that RSB analgesia does not reduce postoperative pain. The effects on catalase as a biomarker of ROS in response to midline laparotomy with RSB analgesia is unknown. Therefore, we decided to compare postoperative pain at rest $\left(\mathrm{NRS}_{\mathrm{r}}\right)$ and under pressure $\left(\mathrm{NRS}_{\mathrm{p}}\right)$ with plasma concentrations of catalase in patients who underwent midline laparotomy randomized into the control group or to one of three active RSB analgesia groups. This changed our study hypothesis to whether the post-surgery placement of the RSB might reduce ROS stress and pain following surgery in patients with benign disease and cancer. Unexpectedly, no differences were detected in the catalase concentrations between the control group and three RSB study groups, thereby also rejecting our second hypothesis that RSB might reduce ROS. No statistically significant differences were detected in the median plasma catalase concentrations between patients with benign diseases and those with cancer postoperatively.
Table IV. The plasma superoxide dismutase 1 (SOD1) and catalase (CAT) marker concentrations in the patients with benign diseases $(n=15)$ and in those with cancer $(n=29)$. Plasma concentrations were measured before (PRE), immediately after (POP1) and 24 hours after (POP2) surgery. Median (interquartile range) concentrations are shown.

\begin{tabular}{llll}
\hline Marker & \multicolumn{1}{c}{ Benign } & Cancer & $p$-Value* \\
\hline SOD1 $(\mathrm{pg} / \mathrm{ml})$ & & & \\
$\quad$ PRE & $167(132-216)$ & $160(107-211)$ & 0.647 \\
POP1 & $182(135-286)$ & $202(136-338)$ & 0.989 \\
POP2 & $105(76-171)$ & $112(86-196)$ & 0.610 \\
CAT $(\mathrm{pg} / \mathrm{ml})$ & & & \\
PRE & $0.62(0.41-1.17)$ & $0.73(0.48-1.30)$ & 0.407 \\
POP1 & $1.09(0.78-2.50)$ & $1.46(1.13-2.36)$ & 0.304 \\
POP2 & $0.83(0.75-1.65)$ & $1.12(0.68-1.50)$ & 0.593 \\
\hline
\end{tabular}

*Mann-Whitney $U$-test.

Purdy reported in an academic thesis that RSB analgesia with repeated doses did significantly enhance patient satisfaction in patients undergoing midline laparotomy (2). The overall satisfaction was better in the repeated-dose group compared with the control group $(p=0.025)$ and oxycodone consumption and plasma oxycodone levels were similar in all four study groups (2). In addition, no serious or unexpected adverse events were reported in that study (2).

We also recently reported that cholecystectomy patients with elevated plasma concentrations of SOD1 and catalase appeared to have significantly lower analgesia requirement during the first 24 hours postoperatively $(3,16)$. In this study, the most important finding was a weak correlation 
between plasma catalase concentrations and pain at rest and under pressure following surgery in patients with benign diseases and cancer. It seems that the midline laparotomy does significantly increase the plasma concentration of catalase, but it is normalized 24 hours postoperatively.

Although the limitation of this study is the small sample size, the patients in the control group had no local anaesthetic use and were blinded using similar wound dressing to that for the patients in the active groups. Therefore, it is unlikely that there is a study bias from the local anaesthetic used or placement of the RSB catheters and it is possible to compare the control group with the active groups.

In conclusion, it is important to develop reliable ROS biomarkers in combination with clinical variables used as predictors of pain and outcome in surgical patients $(17,18)$. The most important finding of the study is a significant correlation between plasma catalase levels and pain at rest and under pressure following surgery in patients with benign diseases and cancer. It seems that the midline laparotomy does significantly increase plasma catalase, but this normalizes quickly. Unexpectedly no differences were detected in the catalase values between the four study groups. In opposition to our original hypothesis, there was no evidence that RSB analgesia reduces postoperative pain.

\section{Conflicts of Interest}

The Authors report no conflicts of interest or financial ties to disclose. The Authors alone are responsible for the content and writing of this article.

\section{Acknowledgements}

The study was funded by the Heikki, Aino and Aarne Korhonen foundation and the EVO-funding of the Kuopio University Hospital, Finland.

\section{References}

1 Sundbom $M$ and Hedberg $\mathrm{J}$ : Use of laparoscopy in gastrointestinal surgery in Sweden 1998-2014: A Nationwide Study. Scand J Surg 106: 34-39, 2017.

2 Purdy M: Rectus Sheath Block After Midline Laparotomy. Publications of the University of Eastern Finland, Dissertations in Health Sciences No 431, 2017.

3 Saimanen I, Kuosmanen V, Kärkkäinen J, Selander T, Aspinen S, Holopainen A, Rantanen T and Eskelinen M: Cholecystectomy patients with high plasma levels of catalase have significantly lower analgesia requirement: a prospective study of two different cholecystectomy techniques with special reference to patients with cancer. Anticancer Res 38: 5417-5422, 2018.

4 Yiannakopoulou ECh, Nikiteas N, Perrea D and Tsigris C: Effect of laparoscopic surgery on oxidative stress response: systematic review. Surg Laparosc Endosc Percutan Tech 23: 101-108, 2013.

5 Olakowski M1, Lampe P, Mekle H and Stefanski L: Changes in activity of antioxidant enzymes in the early period after classical and laparoscopic cholecystectomy. Wiad Lek 50: 213-217, 1997. (Article in Polish, English Abstract).
6 Vodopija N, Ovcak Z, Zupancic M, Korsic L, Kramer F, Krstanoski $\mathrm{Z}$ and Parac I: Tissue ischemia due to $\mathrm{CO}_{2}$ pressure during laparoscopic radical prostatectomy. Coll Antropol 33: 77-82, 2009.

7 Halliwell B: The wanderings of a free radical. Free Radic Biol Med 46: 531-542, 2009.

8 Bauer G: siRNA-based analysis of the abrogation of the protective function of membrane-associated catalase of tumor cells. Anticancer Res 37: 567-581, 2017.

9 Spanidis Y, Goutzourelas N, Stagos D, Kolyva AS, Gogos CA, Bar-Or D and Kouretas D: Assessment of oxidative stress in septic and obese patients using markers of oxidation-reduction potential. In Vivo 29: 595-600, 2015.

10 Robbins D and Zhao Y: Oxidative stress induced by MnSODp53 interaction: pro-or antitumorigenic? J Signal Transduct 2012: 101465, 2012.

11 Santos NP, Pereira IC, Pires MJ, Lopes C, Andrade R, Oliveira MM, Colaco A, Peixoto F and Oliveira PA: Histology, bioenergetics and oxidative stress in mouse liver exposed to $\mathrm{N}$ diethylnitrosamine. In Vivo 26: 921-930, 2012.

12 Purdy M, Kokki M, Anttila M, Aspinen S, Juvonen P, Selander T, Kokki H, Pulkki K and Eskelinen M: Does the post-surgery placement of rectus sheath block analgesia alter the oxidative stress biomarker 8-OHdG concentrations: a randomised trial of patients with cancer and benign disease. Cancer Genomics Proteomics 13: 239-244, 2016.

13 Purdy M, Kokki M, Anttila M, Aspinen S, Juvonen P, Korhonen R, Selander T, Kokki H and Eskelinen M: Does the rectus sheath block analgesia reduce the inflammatory response biomarker IL1RA, IL-6, IL-8, IL-10 and IL-1 $\beta$ concentrations following surgery? A randomised clinical trial of patients with cancer and benign disease. Anticancer Res 36: 3005-3011, 2016.

14 Purdy M, Kärkkäinen J, Kokki M, Anttila M, Aspinen S, Juvonen P, Kokki H, Pulkki K, Rantanen T and Eskelinen M: Does rectus sheath block analgesia alter levels of the oxidative stress biomarker glutathione peroxidase: a randomised trial of patients with cancer and benign disease. Anticancer Res 37: 897-902, 2017.

15 Kärkkäinen J, Selander T, Purdy M, Juvonen P and Eskelinen M: Patients with increased levels of the oxidative stress biomarker SOD1 appear to have dimished postoperative pain after midline laparotomy: a randomized trial with special reference to postoperative pain score (NRS). Anticancer Res 38 : 1003-1008, 2018.

16 Kärkkäinen J, Saimanen I, Selander T, Aspinen S, Harju J, Juvonen $\mathrm{P}$ and Eskelinen M: Gallstone patients with enhanced oxidative stress biomarker SOD1 plasma levels have significantly lower number of postoperative analgesic oxycodone doses: A prospective study with special reference to cancer patients. Anticancer Res 38: 3573-3578, 2018.

17 Velásquez JF, Ramírez MF, Ai DI, Lewis V and Cata JP: Impaired immune function in patients undergoing surgery for bone cancer. Anticancer Res 35: 5461-5466, 2015.

18 Poprac P, Jomova K, Simunkova M, Kollar V, Rhodes CJ, Valko M: Targeting free radicals in oxidative stress-related human diseases. Trends Pharmacol Sci 38: 592-607, 2017.

Received September 18, 2018

Revised October 10, 2018

Accepted October 15, 2018 